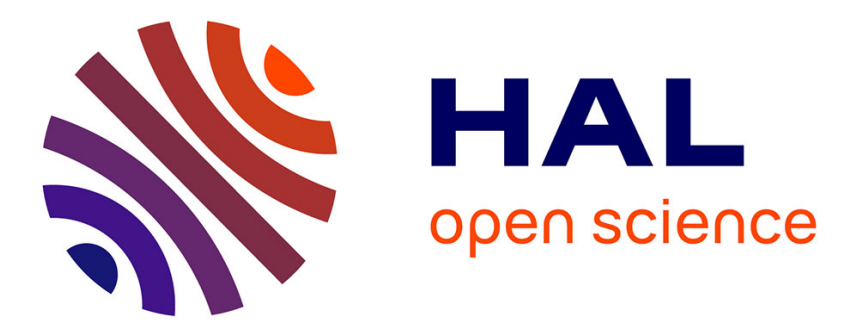

\title{
Architectural performance analysis of FPGA synthesized LEON processors
}

\author{
Corentin Damman, Gregory Edison, Fabrice Guet, Eric Noulard, Luca \\ Santinelli, Jérôme Hugues
}

\section{- To cite this version:}

Corentin Damman, Gregory Edison, Fabrice Guet, Eric Noulard, Luca Santinelli, et al.. Architectural performance analysis of FPGA synthesized LEON processors. 27th International Symposium on Rapid System Prototyping, Oct 2016, Pittsburgh, United States. pp. 33-40, 10.1145/2990299.2990306 . hal-01543282

\section{HAL Id: hal-01543282 \\ https://hal.science/hal-01543282}

Submitted on 20 Jun 2017

HAL is a multi-disciplinary open access archive for the deposit and dissemination of scientific research documents, whether they are published or not. The documents may come from teaching and research institutions in France or abroad, or from public or private research centers.
L'archive ouverte pluridisciplinaire HAL, est destinée au dépôt et à la diffusion de documents scientifiques de niveau recherche, publiés ou non, émanant des établissements d'enseignement et de recherche français ou étrangers, des laboratoires publics ou privés. 


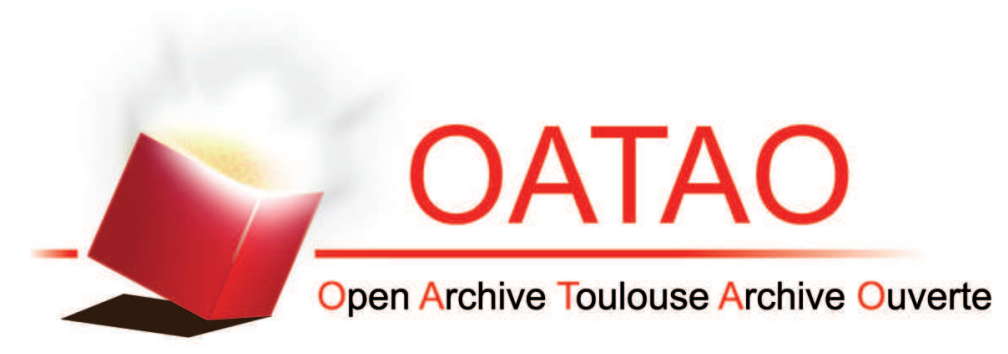

\section{Open Archive Toulouse Archive Ouverte (OATAO)}

OATAO is an open access repository that collects the work of some Toulouse researchers and makes it freely available over the web where possible.

This is an author's version published in: https://oatao.univ-toulouse.fr/17805

Official URL:http://dx.doi.org/10.1145/2990299.2990306

\section{To cite this version :}

Damman, Corentin and Edison, Gregory and Guet, Fabrice and Noulard, Eric and Santinelli, Luca and Hugues, Jérôme Architectural performance analysis of FPGA synthesized LEON processors. (2016) In: 27th International Symposium on Rapid System Prototyping, 1 October 2016 - 7 October 2016 (Pittsburgh, United States).

Any correspondence concerning this service should be sent to the repository administrator: tech-oatao@listes-diff.inp-toulouse.fr 


\section{Architectural Performance Analysis of FPGA Synthesized LEON Processors}

\author{
Corentin Damman \\ Institut Supérieur de \\ l'Aéronautique et de l'Espace \\ (ISAE-SUPAERO) \\ Université de Toulouse \\ 31055 TOULOUSE Cedex 4 \\ corentin.damman@isae.fr \\ Eric Noulard \\ ONERA \\ (The French Aerospace Lab) \\ TOULOUSE \\ eric.noulard@onera.fr
}

\author{
Gregory Edison \\ Institut Supérieur de \\ l'Aéronautique et de l'Espace \\ (ISAE-SUPAERO) \\ Université de Toulouse \\ 31055 TOULOUSE Cedex 4 \\ gregory.edison@isae.fr \\ Luca Santinelli \\ ONERA \\ (The French Aerospace Lab) \\ TOULOUSE \\ luca.santinelli@onera.fr
}

\author{
Fabrice Guet \\ ONERA \\ (The French Aerospace Lab) \\ TOULOUSE \\ fabrice.guet@onera.fr \\ Jerome Hugues \\ Institut Supérieur de \\ l'Aéronautique et de l'Espace \\ (ISAE-SUPAERO) \\ Université de Toulouse \\ 31055 TOULOUSE Cedex 4 \\ jerome.hugues@isae.fr
}

\begin{abstract}
Current processors have gone through multiple internal optimization to speed-up the average execution time e.g. pipelines, branch prediction. Besides, internal communication mechanisms and shared resources like caches or buses have a significant impact on Worst-Case Execution Times (WCETs). Having an accurate estimate of a WCET is now a challenge. Probabilistic approaches provide a viable alternative to single WCET estimation. They consider WCET as a probabilistic distribution associated to uncertainty or risk.

In this paper, we present synthetic benchmarks and associated analysis for several LEON3 configurations on FPGA targets. Benchmarking exposes key parameters to execution time variability allowing for accurate probabilistic modeling of system dynamics. We analyze the impact of architecturelevel configurations on average and worst-case behaviors.
\end{abstract}

\section{CCS Concepts}

$\bullet$ Hardware $\rightarrow$ Statistical timing analysis; $\bullet$ Computer systems organization $\rightarrow$ Embedded hardware; Real-time system architecture; Reconfigurable computing;

\section{Keywords}

Performance Analysis; Embedded Systems; Benchmarking; Processor Synthesis; FPGA; LEON; Probabilistic WorstCase Execution Time

\section{INTRODUCTION}

Timing constraints contribute defining the correctness of most of embedded systems. For example, the processor that manages the injection of an engine or the electronic flight controls of an aircraft have to provide results within welldefined time windows. Such systems are called real-time systems. In particular, hard real-time systems if timing constraints misses are not tolerated and soft-real-time systems if some timing constraints misses can be tolerated.

Recent improvements and optimization of processors induce huge complexity onto real-time systems. We are now facing multi-core architectures with internal communications, cache memories, interruptions, multi-functionalities, etc. Each of which has to be properly analyzed in order to guarantee both system correctness and predictability. Classical single value Worst-Case Execution Time (WCET), based on modeling internal architectures behavior, may have reached its limit. Indeed, complexity in micro-architecture and/or costs in modeling system interactions, may lead to a large pessimism for the worst-case scenarios.

Probabilistic approaches now emerge as alternatives to single value WCETs. The probabilistic real-time modeling assumes the task WCET as worst-case distribution, the probabilistic WCET (pWCET), able to upper-bound any possible task execution behavior. The pWCETs allow accounting for the probability of occurrence of worst-case conditions which could be vanishingly small [6]. Hence, pWCETs may lead to important reduction of capability over-provisioning since they cope better with tasks behavior.

\subsection{Related Work}

Performance analysis of multi-core platforms is an established research field, [13-14]. The approaches proposed so far refer to measurements for evaluating the impact of architectural elements on system average performance, [11-15].

The probabilistic timing analysis estimates pWCETs and can be either static-based or measurements-based. Static Probabilistic Timing Analysis (SPTA) [3-7] requires an exact model of the system in order to infer the probability law, 
thus computing the pWCETs. Measurements-Based Probabilistic Timing Analysis (MBPTA) relies on measurements and on the Extreme Value Theory (EVT) in order to provide $\mathrm{pWCET}$ distributions, [6-8-10]. The MBPTA does not require models of the system.

Contributions : This paper investigates the impacts of CPU architecture elements on tasks execution behaviors. For that purpose we synthesize LEON3 processors with different architectures on two Xilinx FPGAs. We modify some core configuration parameters like cache policy, the branch prediction and the floating-point unit to determine their impact. We run some benchmarks selected from the Mälardalen WCET project [9] on the synthesized architectures. Finally, we analyze the results with the use of a measurement-based probabilistic timing analysis tool called DIAGXTRM [8], to perform average and worst-case performance analysis with probabilistic models ${ }^{1}$.

Organization of the paper: Section 2 and Section 3 present the architectural parameters applied for synthesizing LEON3 processors. Section 4 describes the benchmarks and the task execution conditions applied. Section 5 introduces the DIAGXTRM framework applied for computing the pWCETs and for timing performance analysis. Section 6 presents the results obtained in terms of both average performance and worst-case probabilistic models. Section 7 is for conclusions and future work.

\section{MICROPROCESSOR DESIGN SPACE}

Recent processors architectures rely on multiple building blocks. In the following, we consider those with an impact on the pWCET and present their key parameters.

- Cache memories have a direct impact on performance through instruction/data prefetching. If there is no cache, the system is forced to load each instruction from the memory and to do multiple loads and stores for each variable directly. The consequent saturation of the memory buses will have an impact on the instruction latencies.

- Cache replacement policies control how cache misses are handled, and how new pages are loaded. Different policies may either speed up or slow down a software.

- Branch prediction controls how instructions are prefetched in the pipeline. Such policies usually rely on heuristics.

- Math co-processor enables faster mathematical operations compared to software-emulated ones.

For this study, we implement LEON3 processors, a 32bit SPARC V8 processor, which general architecture is presented in Figure 1.

We synthesize each LEON3 platform configuration using the GAISLER GRLIB VHDL IP LIBRARY written in VHDL, [2], for the GAISLER GR-XC6S-LX75 and the XILINX ML605, which are two boards with XiLinx FPGAs, DDR RAM, Flash, Ethernet, USB, UART, etc. The two FPGAs are flashed with various configurations, according to the four key parameters considered. The architecture and its configuration space are presented in the next subsections.

\subsection{Architecture}

The architecture we consider is based on two LEON3MP processors at $50 \mathrm{MHz}$. We make the following design choices.

\footnotetext{
${ }^{1} \mathrm{~A}$ data pack with all the models is available at https://forge.onera.fr/projects/syntheticbench (login: SyntheticBench and password: anonymous)
}

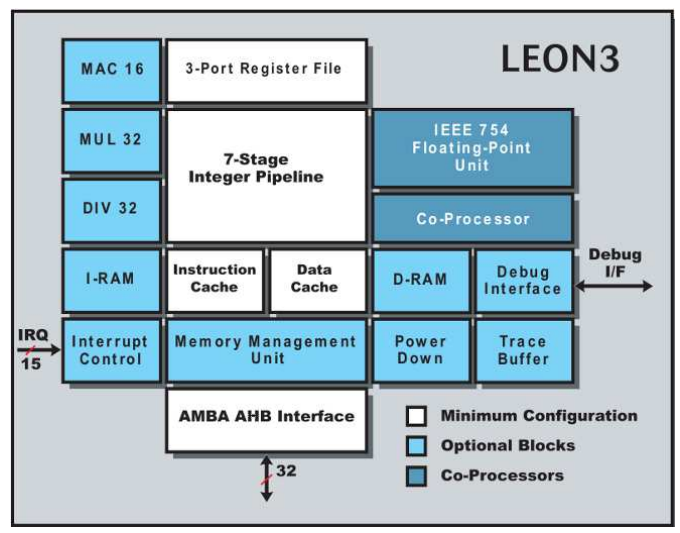

Figure 1: LEON3 Architectural Blocks.

The integer unit is configured with SPARC V8 multiply and divide instructions. Emulated floating-point operations also benefit from this option. The integer multiplier is based on a $32 \times 32$ pipelined multiplier with a two-cycles latency.

For the cache system, LEON3 processors feature two separate instruction and data caches (Harvard architecture) with snooping and locking. The Instruction Cache (IC) is implemented as a multi-way cache with 4 ways, 4 kbytes/way and 32 bytes/line. The Data Cache (DC) is implemented with 4 ways, 4 kbytes/way and 16 bytes/line. Four cache replacement algorithms are proposed: random, direct, LeastRecently-Replaced (LRR) and Least-Recently-Used (LRU). Local instruction and data RAM are disabled. We also choose to disable the Memory Management Unit (MMU).

We implement a hardware module called LEON3 STATISTICAL UNIT (L3STAT). Eight counters are enabled, four per processor: the execution time counter (counted in CPU ticks at a frequency of $50 \mathrm{MHz}$ ), the data and instruction cache miss counters and the branch prediction miss counter. In this work we use the execution time counter for evaluating the impact of each configuration on the task executions.

\subsubsection{Cache Memory}

Cache memories are key elements of embedded systems since, depending on the state of the cache, task execution time could change. Both the worst-case timing analysis and the accuracy of the pWCET estimates would be impacted by the cache. We implement two cases: with caches (caches) and without caches (nocaches). In the first case the cache hierarchy is fully active, while in the second we disabled the twos caches for each processor. We expect a penalty on the execution time from the absence of cache.

\subsubsection{Cache Replacement Policies}

Cache replacement policies impact system performance i.e. task execution time. The way cache lines are replaced would affect next accesses; thus the latency of retrieving the information in cache changes. The replacement policies are critical to system variability especially with benchmarks which saturate the cache. The different replacement algorithms implemented are: the Random algorithm, which selects an item randomly and evicts it; the Direct algorithm, where the address of the new item is directly used to calculate its location in the cache; the $L R R$ policy: it evicts the item least recently replaced; the $L R U$ policy which evicts the item least recently accessed. 


\subsubsection{Branch Prediction (BP)}

The LEON3 is an advanced 7-stage pipelined processor, which implies the use of an efficient BP. According to the GRLIB Configuration Help, the BP option would improve performance with up to $20 \%$, depending on application. In order to verify that gain of performance and measure the impact of this option on the worst-case behavior, we run the benchmarks on a platform with branch prediction $(B P)$ and without branch prediction ( $n o B P$ ) for comparison.

\subsubsection{Floating-Point Unit (FPU)}

The FPU is a high-performance, fully pipelined IEEE754 FPU. The two FPUs provided are the GaISLER RESEARCH'S GRFPU and GRFPU-LITE. The GRFPU is a high-performance pipelined FPU with high area requirements. GRFPU-LITE provides a balanced option with high acceleration of floating-point computations combined with lower area requirements compared to GRFPU. They support all SPARC FPU instructions. If the FPU is disabled, a simulated, software FPU can be used. The option without FPU is identified noFPU, while that with FPU are identified as GRFPU or GRFPU-lite.

\subsection{Architectural Configurations}

A platform configuration is made of a set of architectural elements: caches, cache replacement policies, BP and FPU. We define the reference configuration as Reference $=$ (caches, Random (IC), Random (DC), BP, noFPU). In the following, Reference indicates the choice on the element composing the reference configuration. All the other configurations are compared with Reference.

Other configurations considered are:

Direct $=($ caches, Direct (IC), Direct (DC), BP, noFPU), $\mathrm{LRR}=($ caches, LRR (IC), LRR (DC), BP, noFPU), $\mathrm{LRU}=($ caches, $L R U(\mathrm{IC}), L R U(\mathrm{DC}), B P$, noFPU $)$, noBP $=($ caches, Random (IC), Random (DC), noBP, noFPU), nocaches $=($ nocaches, N/A, N/A, BP, noFPU), $\mathrm{GRFPU}=$ (caches, Random (IC), Random (DC), BP, GRFPU), GRFPU-lite $=$ (caches, Random (IC), Random (DC), BP, GRFPU-lite)

The configuration of a typical System on a Chip (SoC) architecture like the GAISLER GR712RC is GR712RC $=($ caches, $L R U$ (IC), $L R U$ (DC), BP, GRFPU), [1]. We will consider each choice of this architecture.

\section{MICROPROCESSOR SYNTHESIS}

The first step of this work is to synthesize the different architectural configurations presented in Section 2. Figure 2 shows the entire methodology with the steps considered, respectively for synthesizing, benchmarking and analyzing.

We use the GR-XC6S board for the architectural configurations Reference, Direct, LRR, LRU, noBP and nocaches; we use the ML605 board instead for the configurations GRFPU and GRFPU-lite to overcome limits in synthesizing FPUs. The GR-XC6S is a SPARTAN-6 FPGA, the ML605 board feature a Virtex-6 FPGA [19-20].

Figure 4 shows the occupations of the FPGAs in terms of slice registers (mostly Flip Flops), slice Look-Up Tables (LUTs, mostly used as logic) and the global numbers of occupied slices. The Table 1 presents detailed values.

We compare the different architectures related to Reference. We note that the number of occupied slices for the Reference configuration is not the same for both FPGAs:
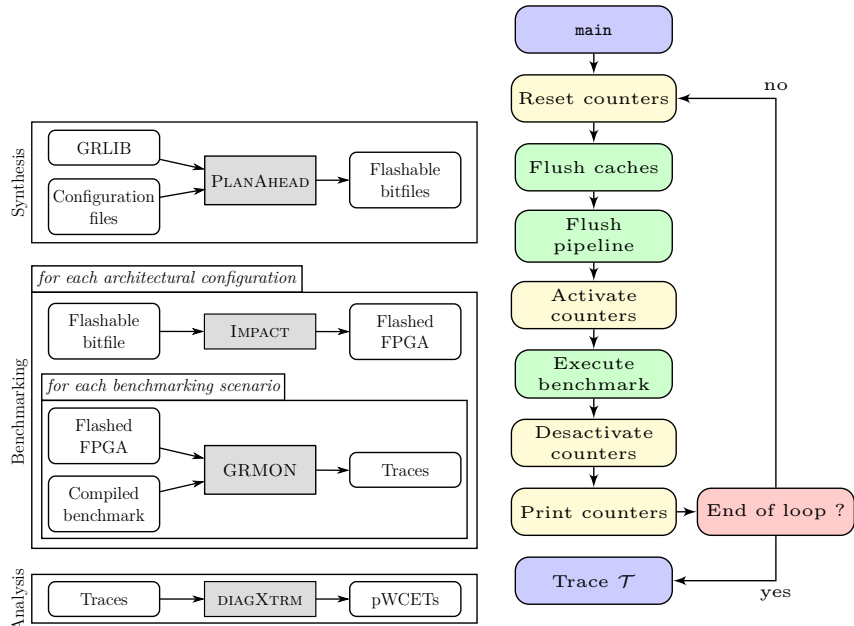

Figure 2: Methodology applied.

\section{Figure 3: Bench- marking execution diagram.}

this is due to the differences between Spartan-6 and Virtex6 internal architectures. Moreover, many slices are used for communication with external elements on the ML605 board that the GR-XC6S does not have.

- Removing the data and instruction caches reduce slices by $14.81 \%$ and run-time performances, see Section 6 .

- Architectures using the Direct or the LRR replacement algorithm uses almost the same slices as the Random policy. However, the LRU policy occupies $32.11 \%$ more space. This area overhead is due to the addition of 5 flip-flops per line (for a 4-way LRU) to store the accesses.

- Disabling the branch prediction only saves $6.73 \%$ of the number of occupied slices, and reduces the performance.

- The implementation of the FPU on the ML605 board shows that the GRFPU uses almost twice the slices required without FPU. The GRFPU-LITE is, on the other hand, less demanding in terms of occupied slices.

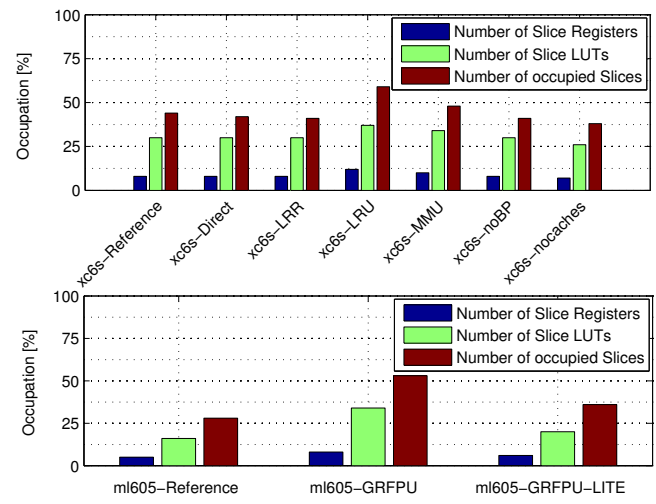

Figure 4: Device utilization summary.

Table 1: Device Utilization Summary.

\begin{tabular}{|c|c|c|c|}
\hline $\begin{array}{r}\text { GR-XC6S } \\
\text { architecture }\end{array}$ & $\begin{array}{l}\text { Occupied slices } \\
\text { (out of 11662) }\end{array}$ & $\begin{array}{r}\text { ML605 } \\
\text { architecture }\end{array}$ & $\begin{array}{l}\text { Occupied slices } \\
\text { (out of } 37680 \text { ) }\end{array}$ \\
\hline Reference & $5219 \Rightarrow$ & Reference & $10667 \Rightarrow$ \\
\hline Direct & $4957 \Rightarrow$ & GRFPU & $20198 \Rightarrow+89.35 \%$ \\
\hline LRR & $4791 \Rightarrow$ & GRFPU-lite & $13724 \Rightarrow+28.66 \%$ \\
\hline LRU & $6895 \Rightarrow+32.11 \%$ & & \\
\hline noBP & $4868 \Rightarrow-6.73 \%$ & & \\
\hline nocaches & $4446 \Rightarrow-14.81 \%$ & & \\
\hline
\end{tabular}




\section{EMBEDDED SYSTEM BENCHMARKING}

We chose the Mälardalen WCET benchmark programs to implement tasks and to push the processor to its limits and to reveal the impact of each architectural change on both average and worst-case execution time of tasks.

- cnt and matmult are used to fill entirely the data cache with matrix operations. cnt counts non-negative numbers in a matrix with a parametrized size of $1000 \times 1000$. matmult performs a matrix multiplication. As for cnt, the size is parameterizable and we used $200 \times 200$ matrix. Those benchmarks measure the performance of the cache replacement policy and the impact of the (lack of) caches.

- nsichneu is similar to the first two benchmarks, but for the instruction cache. It uses a large amounts of ifstatements (more than 250) to simulate an extended Petri Net. The benchmark has three variants, nsichneu_inner is considered because it generates the most variability.

- jfdctint is a typical application used in embedded realtime systems. By typical application we mean an application that does not saturate neither the data cache nor the instruction cache. jfdctint performs a discrete cosine transform on a $8 \times 8$ pixel block. The code is made of long calculation sequences (i.e., long basic blocks) and single-nested loops.

- lms is used for FPU performances comparison. The benchmark realizes a Least Mean Squares (LMS) adaptive signal enhancement on a sine wave with added white noise.

All benchmarks are single path tasks, and so no functional variability (input vector) is considered. Also there is no need to look for the worst case path.

We use GRMON monitor from Gaisler. It allows a nonintrusive debugging of LEON3 systems. It also performs communication with the modules implemented in the architecture, like the L3STAT unit. Finally, it is used to download and run applications on FPGAs.

\subsection{Execution Conditions}

The benchmarks are executed on all architectural configurations, with medium optimization and SPARC V8 instructions. We investigate two execution conditions.

The first execution condition is to run the task alone, without any other interaction. This execution condition is the baseline, as it is exempt of any interference tasks by construction. All benchmarks were compiled for this execution condition, and the flag -msoft-float was used to emulate floating-point operations when no FPU was present. This execution condition is named ELF after its compiler name.

The second execution condition consider using the RTEMS Real-Time Operating System (RTOS), [17]. This execution condition is more representative of an actual real-time embedded system. It is compiled in Asymmetric Multi Processor (AMP) mode, to exercise perturbations between cores. This execution condition is identified as RTEMS.

The RTEMS Multi-Processor application is running in the pre-emptive mode on two cores. In the first core, two tasks are launched: the benchmarking task with priority 2 and a second interference task, doing infinite loops, with priority 1 (higher priority). The second core contains another task made of an infinite loop. This minimal intra- and intercore interference is used to determine the effect of interactions and is representative of a not heavily loaded system. The methodology could apply to any kind of interference, and it will be considered in future work.

\subsection{Initial State Assumptions}

The benchmarking execution diagram is presented in Figure 3. The trace is made of 5000 consecutive executions of the same benchmark. There exists a trace per benchmark, per configuration and per execution condition.

We must ensure that the processor is in the same initial state at each benchmark execution. Before each run, the hardware counters from the L3STAT unit are reset, the caches are flushed and the pipeline is filled with nop() instructions. Then, the counters are activated, the benchmark is executed and finally the eight counters are disabled and printed for that execution. The resulting trace $\mathcal{T}$ is a matrix of size $8 \times 5000$ (eight counters, measured for each of the 5000 executions). The execution time is measured in CPU ticks and the trace duration spans from 4 minutes to 30 hours, depending on the benchmark and the architectural configuration. This allows including multiple systemic effects in the traces which could happen late in the executions.

\section{PROBABILISTIC PERFORMANCE EVAL- UATION}

The traces obtained from the benchmarking traces are applied to estimate the pWCETs, Figure 2.

Measurements-Based Probabilistic Time Analysis (MBPTA) allows defining tasks execution behaviors from runtime measurements. We use the MBPTA approach called DIAGXTRM to evaluate the impacts of architectural elements on both average and worst-case behavior of tasks. Only the execution time counter from the L3STAT traces is used.

The Extreme Value Theory (EVT) is the statistical tool composing any MBPTA that produces continuous distributions which are safe estimation of the task worst-case behavior $^{2}$ : the pWCETs. For this specific paper, DIAGXTRM applies the EVT in its peak over threshold version identifying the pWCETs from the Generalized Pareto Distribution (GPD) family. The distribution shape parameter $\xi$ identifies the distribution that better cope with the measurements within this family. In particular, $\xi<0$ is for a Weibull distribution, $\xi=0$ is for a Gumbel distribution and $\xi>0$ is for a Frechet distribution.

Hence, the pWCET is a worst-case thresholds with a probability associated to the risk to go past this value.

The EVT, thus the MBPTA, is applicable if the measurements are 1 . stationary, 2 . independent or 3 . extremal independent and 4 . they match a specified theoretical model, Figure 5. These situations correspond to four hypothesis that can be asserted from measurements. DIAGXTRM verifies all those hypotheses with the corresponding statistical tests. The confidence on the hypotheses validation is related to the quality of the pWCET model produced, named reliability of the pWCET. Only if all the tests succeed, the pWCET obtained is reliable and safe. To note that the independence and the extremal independence are partially overlapping hypotheses; if one is verified, there is no need to verify the other, Figure 5.

The robust statistics [5-8] is applied by DIAGXTRM for quantifying the uncertainties from statistical tests. It defines the confidence $c l$ on the hypothesis verified.

\footnotetext{
${ }^{2}$ By safe estimation we mean a $p W C E T$ which is larger than or equal to any possible task execution time, [7].
} 


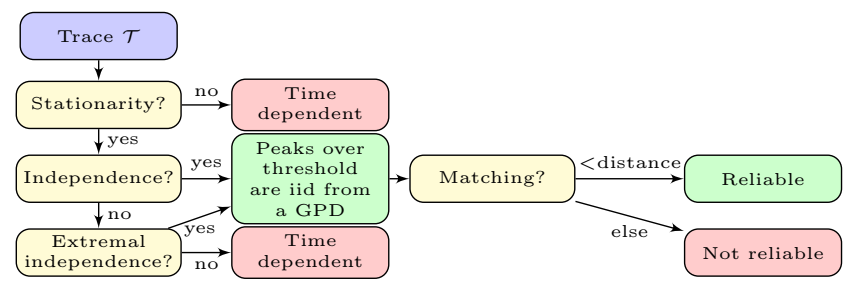

Figure 5: Decision diagram of diagXtrm with tests and action applied.

Stationarity Hypothesis: The analysis of trace can show the relationship between consecutive measurements and evaluate the impact that previous (in time) measurements on future ones, i.e. their independence. The EVT applicability relates to stationary traces and is measured by the The Kwiatowski Phillips Schmidt Shin test, [12] as $c l_{1}$.

INDEPENDENCE HYPOTHESIS: The statistical dependence is checked through the existence of correlated patterns of measurements, using the Brock Dechert Scheinkman statistical test [4]. The confidence on the independence is $c l_{2.1}$.

EXTREMAL INDEPENDENCE HYPOTHESIS: When overall independence does not hold, another way is to look for the independence of extreme measurements ${ }^{3}$. The extremal in$\operatorname{dex} \theta \in[0,1]$ is an indicator of dependence degree between extreme measurements, [16]. We denote this parameter $c l_{2.2}$.

MATChing Hypothesis: The matching test is based on a quadratic statistic which measures the square distance between the pWCET model estimated and an hypothetical exact pWCET model following the GPD. The Cramer Von Mises criterion is applied for verifying the degree of matching between the two distributions, leading to parameter $\mathrm{Cl}_{3}$.

Finally, DIAGXTRM computes pWCET parameters such as the threshold $u$ and the pWCET distribution shape $\xi$ that guarantee the best pWCET model among the possible EVT pWCETs, see [8] for details.

We stress the fact that with MBPTA approaches, the resulting pWCET model represents the worst-case for the execution conditions accounted for by the measurements. It is not an absolute worst-case. Thus safety, confidence and reliability relates to the specific measurements considered.

\section{TRACE ANALYSIS}

Given a trace of measurements, DIAGXTRM profiles both the average and the worst-case behavior of tasks. We use that tool for each architectural configuration and present the results in the following subsections.

\subsection{Average Performance}

At first we focus on the average execution time to compare the architectural configuration effects.

\subsubsection{Execution Time Variability}

Figure 6 illustrate the execution time variability of any system. More precisely, the trace is the result of 5000 executions of the cnt benchmark on the reference architecture without any RTOS. That proves that even when an application is run in a standalone mode, variability is present due to systemic and functional changes happening at runtime, due to caches, BP, etc.

\footnotetext{
${ }^{3}$ By extremal measurements we allude to observations relatively far from the average values as well as observations well separated in time.
}

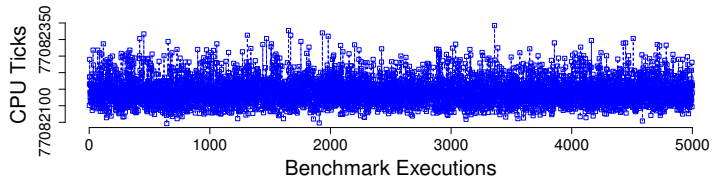

(a) Time trace of execution times.

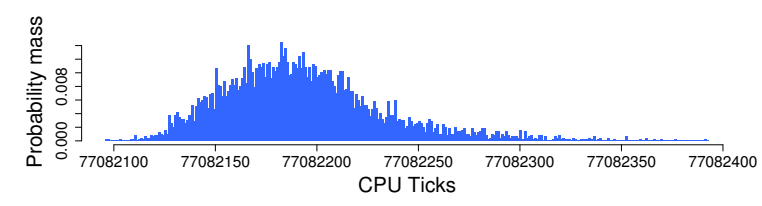

(b) Histogram of execution times.

Figure 6: Time trace and histogram of execution times (benchmark cnt on the GR-XC6S reference architecture without RTEMS).

\subsubsection{Impact of the Cache Replacement Policy}

We analyze the traces of the two benchmarks matmult and nsichneu which saturate respectively the data and the instruction caches.

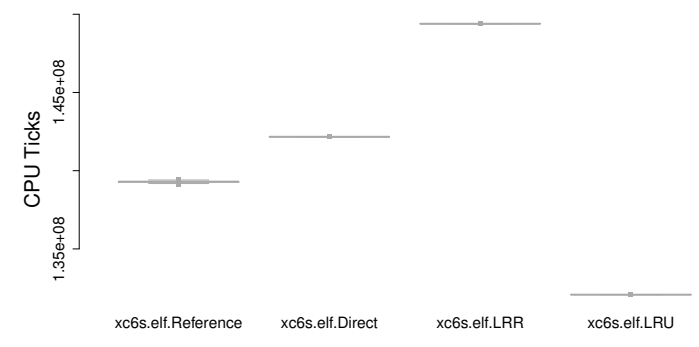

(a) matmult on GR-XC6S without RTEMS.

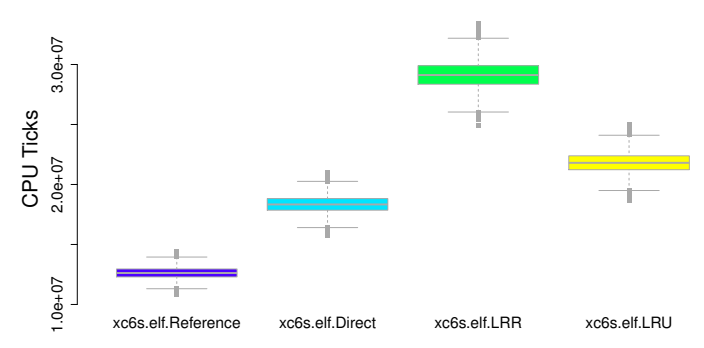

(b) nsichneu on GR-XC6S without RTEMS.

Figure 7: Box plots of execution times for different cache replacement policies.

Figure 7a presents the box plots of execution times for the matmult benchmark. We can see that in order to improve the average performance of the data cache, the best cache replacement policy is the LRU algorithm. Then comes the random, the direct and finally the LRR algorithm.

As a matter of fact, programs usually compute results thanks to a restricted set of variables. It is then natural that the LRU algorithm is the best choice for the data cache replacement policy. This result is consistent with the fact that multiple SoC architectures implement the LRU algorithm for their data cache replacement policy, like the GR712RC configuration. 
Figure $7 \mathrm{~b}$ illustrates the execution times as box plots for the benchmark nsichneu. We can see that the LRU algorithm is not the best replacement policy for the instruction cache. The random algorithm is the first, then the direct, the LRU and finally the worst is the LRR.

For instruction cache the random replacement policy has better performance on average than the other policies, [3]. Unlike data variables accesses, sequences of instructions of any program are not really regular. That explains why the random algorithm is the best instruction cache replacement policy. However, the choice of another algorithm is not critical as the drawback induced on the execution time is not significant, comparatively to the bigger differences of execution times related to the data cache replacement policies, Figure $7 \mathrm{a}$ (to note that the scales are not the same in the two figures).

\subsubsection{Disabling Key Architectural Elements}

In order to evaluate the gain of performance provided by the branch prediction or the cache memories, we disabled them from reference architecture. The Table 2 summarizes the execution time distributions and the performance drawbacks for different benchmarks. We chose matmult and nsichneu because they overuse the cache memories when present. Thus, those benchmarks are prone to reveal the catastrophic effects of disabling the instruction and the data caches. The third benchmark analyzed is jfdctint which is applied for investigating the impact on the execution time for an application that does not saturate caches.

Table 2: Execution Time Distributions.

(a) Benchmark matmult on the GR-XC6S without RTEMS.

\begin{tabular}{r|rrr|r}
$\begin{array}{r}\text { GR-XC6S } \\
\text { architecture }\end{array}$ & \multicolumn{2}{|c|}{ Execution Time Distribution } & Perf. \\
\hline Reference & 139147148 & \multicolumn{1}{c}{ Mean } & Max & drawback \\
noBP & 154722839 & 1548692184 & 139447778 & - \\
nocaches & 1095326983 & 1095468302 & 155006999 & $11.19 \%$ \\
& & & & 68562181
\end{tabular}

(b) Benchmark nsichneu on the GR-XC6S without RTEMS.

\begin{tabular}{r|crr|r} 
GR-XC6S & \multicolumn{2}{|c|}{ Execution Time Distribution } & Perf. \\
architecture & Min & \multicolumn{1}{c}{ Mean } & \multicolumn{1}{c}{ Max } & drawback \\
\hline Reference & 10828017 & 12624121 & 14445302 & - \\
noBP & 11597909 & 13516958 & 15485162 & $7.20 \%$ \\
nocaches & 94652781 & 110600474 & 126897285 & $778.47 \%$
\end{tabular}

(c) Benchmark jfdctint on the GR-XC6S without RTEMS.

\begin{tabular}{r|rrr|r} 
GR-XC6S & \multicolumn{2}{|c|}{ Execution Time Distribution } & Perf. \\
architecture & \multicolumn{1}{|c|}{ Min } & Mean & Max & drawback \\
\hline Reference & 2468750 & 2468762 & 2468788 & - \\
noBP & 2546749 & 2546760 & 2546791 & $3.16 \%$ \\
nocaches & 16326625 & 16328010 & 16329192 & $561.43 \%$
\end{tabular}

As described in Section 2, the branch prediction deteriorates performances up to $20 \%$ if disabled. A maximal drawback of $11.19 \%$ is measured, Table 2 a.

The cache memories impact on the execution time distribution is huge. For the same application, a factor of 8.78 on the execution time can be observed (Table $2 \mathrm{~b}$ ), where a factor of at least 2-3 was expected according to the GRLIB Configuration Help. For a typical application, the performance drawbacks are not negligible, Table 2c.

The results demonstrate that branch prediction and cache memories are key elements for embedded systems. Although they clearly improve average performances, they increase execution time variability. The system predictability or analyzability are affected by those elements.

\subsubsection{Impact of The FPU Architecture}

We use the benchmark $l m s$ that computes a lot of floatingpoint operations. Figure 8 presents the first 1000 executions of the resulting traces. We note that there are different execution modes, each with some variability, depending on the FPU architecture. The only element which is not reset between each benchmark execution is the FPU branch prediction, as the integer unit branch prediction and the cache memories are flushed each time. The multiple modes are then probably due to a non-zero prediction distance as detailed in [18].

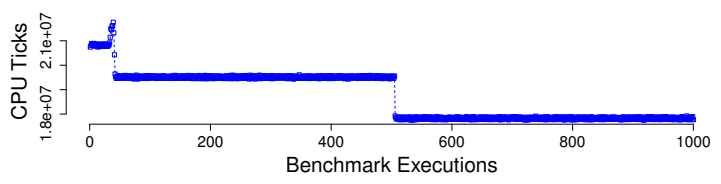

(a) Reference architecture with software FPU.

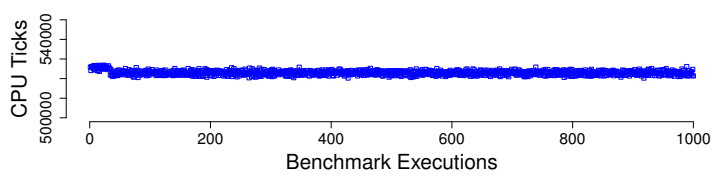

(b) Architecture with GRFPU.

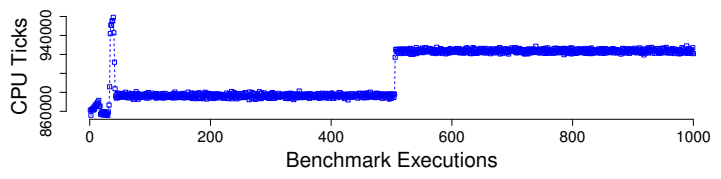

(c) Architecture with GRFPU-LITE.

Figure 8: Time traces of execution times (lms on the ML605 without RTEMS).

In the remaining 4000 measurements only one execution mode exists, revealing a convergence to a mode after some executions. Its mean value is used for comparison between the 3 FPUs. The emulated FPU has a mean level of $17^{\prime} 826^{\prime} 717$ CPU ticks, the GRFPU converges at $522^{\prime} 888$ CPU ticks and the GRFPU-LITE at $923^{\prime} 719$ CPU ticks. Improvements are (respectively) of $33 \times$ and $18 \times$ faster.

The advantages of using a hardware FPU instead of an emulated FPU for floating-point applications are comforted; GRFPU is up to 34 times faster than a software FPU; GRFPU-LITE is a good compromise between speed improvement (19 times faster) and occupied slices.

A side comment regards the capability that DIAGXTRM has of modeling architectural elements without the need for an exact element model. For example, with $l m s$ and the FPU architecture, although not knowing the internal behavior of the FPUs applied, DIAGXTRM is able to characterize the different modes just from measurements.

Table 3 summarizes the average execution time of each architectural change. This is done for the five benchmarks of interest and for each execution condition.

Two additional configurations were synthesized: the yare identified as Best. For the GR-XC6S it is Best $1=($ caches, Random (IC), LRU (DC), BP, noFPU) and for the ML605 it is Best $2=$ (caches, Random (IC), LRU (DC), BP, GRFPU). They are the best configuration since they collect the choices which improves the most the average performances, respectively for each FPGA architectures. 


\begin{tabular}{|c|c|c|c|c|c|c|c|c|c|c|c|c|}
\hline \multirow{2}{*}{\multicolumn{2}{|c|}{$\begin{array}{c}\text { Embedded system } \\
\text { benchmarking }\end{array}$}} & \multicolumn{11}{|c|}{ Table 3: Mean execution time in thousands of CPU ticks. } \\
\hline & & \multicolumn{4}{|c|}{$\begin{array}{c}\text { GR-XC6S Archi } \\
\text { Cache Replacement Policies }\end{array}$} & \multicolumn{2}{|c|}{ Key Elements } & \multirow{2}{*}{$\begin{array}{l}\text { Best } \\
\text { Best1 }\end{array}$} & & FPU & & Best \\
\hline Env. & Bench. & Reference & Direct & LRR & LRU & noBP & nocaches & & Reference & GRFPU & GRFPU-lite & Best2 \\
\hline \multirow{5}{*}{ ELF } & cnt & 77.082 & 77.082 & 77.082 & 77.082 & 81.132 & 436.601 & 77.082 & 77.276 & 77.276 & 77.276 & 77.276 \\
\hline & matmult & 139.292 & 142.168 & 149.393 & 132.077 & 154.869 & 1095.468 & 132.077 & 129.721 & 129.721 & 129.721 & 129.721 \\
\hline & nsichneu & 12.624 & 18.337 & 29.122 & 21.806 & 13.517 & 110.600 & 12.624 & 21.638 & 21.791 & 21.785 & 12.627 \\
\hline & jfdctint & 2.469 & 2.469 & 2.469 & 2.469 & 2.547 & 16.328 & 2.469 & 2.501 & 2.485 & 2.485 & 2.501 \\
\hline & lms & 17.738 & 17.737 & 18.312 & 17.737 & 19.108 & 178.854 & 17.738 & 17.827 & 0.523 & 0.924 & 0.524 \\
\hline \multirow{2}{*}{ RTEMS } & jfdctint & 2.496 & 2.498 & 2.503 & 2.495 & 2.575 & 18.268 & 2.496 & 2.530 & 2.532 & 2.532 & 2.532 \\
\hline & lms & 17.980 & 18.032 & 18.304 & 17.977 & 19.387 & 207.048 & 17.979 & 18.078 & 0.535 & 0.939 & 0.535 \\
\hline Glob: & me scores ${ }^{4}$ & - & $+8.32 \%$ & $+24.58 \%$ & $+11.67 \%$ & $+6.62 \%$ & $+663.63 \%$ & $-1.01 \%$ & - & $-19.35 \%$ & $-18.91 \%$ & $-27.71 \%$ \\
\hline
\end{tabular}

On a global scale, the Best configurations improve the performance more than they deteriorate it, Table 3 . The architectures without BP or without caches are clearly the worst. The LRU algorithm is not always the faster, as we can see with the nsichneu benchmark, but its choice rather than the random replacement policy for the data cache is profitable. As we can see, Best configurations allows an additional gain of $1.01 \%$ on the GR-XC6S and of $8.36 \%$ on the ML605 in comparison with the second best architectures, respectively Reference and GRFPU.

Finally, the effect of the RTOS can be observed: the mean execution times undergo a penalty of $2.52 \%$ on average with RTEMS as compared to standalone ELF.

\subsection{Worst-Case Performance}

This section focuses on the worst-case performance and the estimation of the pWCETs. The methodology applied as well as the quality of the pWCET models (reliability and hypothesis confidence) are detailed. Two cases are investigated: the impact of different cache replacement policies and the impact of the RTOS on worst-case behaviors.

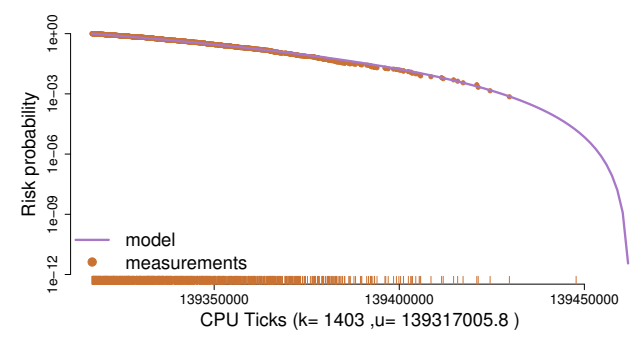

Figure 9: Measurements and model for pWCET estimation (matmult on GR-XC6S without RTEMS).

\subsubsection{Probabilistic Distribution Fitting}

Figure 9 shows the measurements and the pWCET derived from the EVT. The inverse cumulative distributions are represented. $u$ is the threshold parameter that DIAGXTRM computes for obtaining the best pWCET model; $k$ is the number of measurements above the threshold selected. We see how the pWCET model is able to perfectly cope with the input measurements and then safely infers the rare events (risk probability $\leq 10^{-9}$ ).

\subsubsection{Quality of the Probabilistic WCET Model}

As described in Section 5, the reliability of the pWCET model is based on the confidence levels of the four hypotheses $\left\{c l_{1}, c l_{2,1}, c l_{2,2}, c l_{3}\right\}$. Each hypothesis is scored out of 4.0,

\footnotetext{
${ }^{4}$ For each benchmark, a score is determined as $\frac{\text { executionTime }}{\max (\text { executionTimes })}$. Then, the sum of the scores are compared relatively to the Reference.
}

and a minimum of 1.0/4.0 is required in order to apply the method. Hypotheses $c l_{2.1}$ and $c l_{2.2}$ are covering each other, so that a score of $1.0 / 4.0$ in one of the two is sufficient. The spider net in Figure 10 represents the reliability of the pWCET model from these confidence levels.

Hence, all the assumptions are satisfied, the model is reliable. Stationarity, independence, extremal independence and matching are verified with almost the maximal confidence. The EVT is applicable with realistic architectures as there is enough variability from the measurements.

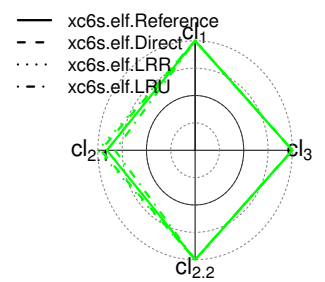

(a) Cache replacement policies (matmult on GR-XC6S without RTEMS).

\section{Figure 10: Reliability of the pWCET estimations}

\subsubsection{Comparisons of Worst-Case Behaviors}

From the pWCET distributions we can extract the WCET thresholds with a $10^{-9}$ risk probability i.e. confidence. This allows us to identify the impact of each architectural change on the worst-case behaviors with single values. Table 4 indicates the pWCET distribution shape parameter $\xi$ for each pWCET model as well as the theoretical estimations i.e. the WCET thresholds at $10^{-9}$. DIAGXTRM automatically selects $\xi$ to best fit the input measurements. As it can be seen, the measurements have small variance hence they are better approximated with a Weibull GPD distribution, $\xi<0$.

The accuracy of the pWCET defines how close the pWCET is to the maximum measured execution time: it is the normalized difference between the pWCET and the highest measured value. As the models are all Weibull distributions, thus bounded to the right, they are quite close to the maximum measured values, Table 4 . The small accuracy indicates that the measured values embed already the worst-case conditions. Thus, the pWCET as Weibull distribution is able to perfectly cope with the measurements and the task average behavior. The case with accuracy equal to $1.32 \mathrm{E}-01$, Table $4 \mathrm{ELF}$, indicates that the measurements have large variability. This means that the worst-case conditions could have a significant impact on the task execution times. The resulting Weibull, in order to account for that 
and foreseen the worst-cases, has to differ more from the measurements. Nonetheless, the pWCET is equally reliable due to the maximum confidences achieved.

The impact of the cache replacement policy on the worstcase behavior is presented in Table 4a. The results are consistent with those presented in Section 6.1.2.

The benchmark $l m s$ was used to characterize the impact of the RTOS. We noted that the behavior of the FPU is not stable in the first 1000 runs of the benchmark, thus we use the last 4000 iterations to determine the pWCET distribution (i.e. achieve the hypotheses scores presented before in Figure 10b). The Table $4 \mathrm{~b}$ gives the results. They confirm that the use of a RTOS like RTEMS induces a small penalty of $2.85 \%$ on the task performance: minimal interference were added and then accounted by a more pessimistic pWCET.

Table 4: pWCET distribution parameters.

(a) Impact of the cache replacement policy

(benchmark matmult on the GR-XC6S without RTEMS).

\begin{tabular}{r|c|ccc} 
GR-XC6S & Distribution & \multicolumn{3}{|c}{ diagXtrm results } \\
architecture & shape $\xi$ & Theoretical & Measured & Accuracy \\
\hline Reference & -0.249 & 139447252.58 & 139433822 & $9.63 \mathrm{E}-03$ \\
Direct & -0.219 & 142170799.12 & 142170390 & $2.88 \mathrm{E}-04$ \\
LRR & -0.095 & 149397501.05 & 149396595 & $6.06 \mathrm{E}-04$ \\
LRU & -0.317 & 132076829.57 & 132076819 & $8.00 \mathrm{E}-06$
\end{tabular}

(b) Impact of the RTOS (benchmark lms on the ML605).

\begin{tabular}{r|c|ccc}
$\begin{array}{r}\text { ML605 } \\
\text { with GRFPU }\end{array}$ & $\begin{array}{c}\text { Distribution } \\
\text { shape } \xi\end{array}$ & \multicolumn{3}{|c}{$\begin{array}{c}\text { diagXtrm results } \\
\text { Theoretical }\end{array}$} \\
\hline ELF & -0.149 & 527244.18 & 526547 & $1.32 \mathrm{E}-01$ \\
RTEMS & -0.596 & 542279.08 & 542228 & $9.42 \mathrm{E}-03$
\end{tabular}

\section{CONCLUSION AND FUTURE WORK}

In this work, we review i) configuration space of LEON processors on FPGA and ii) benchmarking LEON3 processors elements. Some architectural choices have been made, described and then synthesized on two FPGA platforms. Tasks benchmarks have been selected for their ability of revealing the impact of each configuration on average and worst-case behaviors. Execution conditions have been chosen in order to expose key parameters in execution time variability in both standalone and RTOS environments.

The results verify that time execution variability is present in any modern processor. Average performance analyses show that the best data cache replacement policy is the LRU algorithm, while the random algorithm is the best choice for the instruction cache. The results confirm that cache memories, branch predictions and high-performance math co-processor are key elements of modern processors. They contribute in improving average performance at the cost of increased variability in the system.

Probabilistic models have been estimated to show the impact of different cache replacement policies and the impact of the RTOS on the worst-case task behaviors. We validated the reliability and the accuracy of the resulting pWCETs.

This approach could be applied with other benchmarks or architectural configurations. Future work will study interference among tasks in RTOS, and evaluate the impact of scheduler or resource sharing at software and hardware levels (cache, MMU, etc.). This will improve the understanding of modern processors so as to guarantee timing constraints.

\section{ACKNOWLEDGMENTS}

The authors thanks Thales Avionics for their support through the ARISE Chair.

\section{REFERENCES}

[1] Gaisler. GR712RC User's Manual. pp. 43-44, 2016.

[2] Gaisler. GRLIB VHDL IP Core Library, 2016.

[3] S. Altmeyer, L. Cucu-Grosjean, and R. I. Davis. Static probabilistic timing analysis for real-time systems using random replacement caches. Real-Time Systems, 51(1):77-123, 2015.

[4] W. A. Brock, J. A. Scheinkman, W. D. Dechert, and B. LeBaron. A Test for Independence based on the Correlation Dimension. Econometric Reviews, 15(3):197-235, 1996.

[5] J. J. Buckley. Fuzzy statistics: hypothesis testing. Soft Comput., 9(7):512-518, 2005.

[6] L. Cucu-Grosjean, L. Santinelli, M. Houston, C. Lo, T. Vardanega, L. Kosmidis, J. Abella, E. Mezzetti, E. Quiñones, and F. J. Cazorla. Measurement-based probabilistic timing analysis for multi-path programs. In ECRTS, pages 91-101, 2012.

[7] R. I. Davis, L. Santinelli, S. Altmeyer, C. Maiza, and L. Cucu-Grosjean. Analysis of probabilistic cache related pre-emption delays. ECRTS, 2013.

[8] F. Guet, L. Santinelli, and G. Morio. On the reliability of the probabilistic worst-case execution time estimates. In ERTSS, 2016.

[9] J. Gustafsson, A. Betts, A. Ermedahl, and B. Lisper. The Mälardalen WCET benchmarks - past, present and future. pages 137-147, 2010.

[10] J. Hansen, S. Hissam, and G. A. Moreno. Statistical-Based WCET Estimation and Validation. In $W C E T$, pages 1-11, 2009.

[11] W. Jiang, Z. Guo, Y. Ma, and N. Sang. Measurement-based research on cryptographic algorithms for embedded real-time systems. Journal of Systems Architecture, 59(10):1394-1404, 2013.

[12] D. Kwiatkowski, P. C. B. Phillips, P. Schmidt, and Y. Shin. Testing the null hypothesis of stationarity against the alternative of a unit root : How sure are we that economic time series have a unit root? Journal of Econometrics, 54(1-3):159-178, 001992.

[13] D. Mattsson and M. Christensson. Evaluation of synthesizable cpu cores. Master's thesis, Department of Computer Engineering, Chalmers University, 2004.

[14] I. Mhadhbi, N. Rejeb, S. B. Othmen, and S. B. Saoud. Performance Evaluation of FPGA Soft Cores Configurations Case of Xilinx MicroBlaze.

International Journal of Computer Science, Communication $\&$ Information Technology (CSCIT), 1:14-19, 2014.

[15] R. Njuguna. A survey of FPGA benchmarks. Project Report, November, 24, 2008.

[16] P. J. Northrop. An efficient semiparametric maxima estimator of the extremal index. Extremes, 18(4):585-603, 2015.

[17] OAR Corporation. RTEMS, An Open Real-Time Operating System. http://www.rtems.com, 2016.

[18] G. Tyson. The effects of predicated execution on branch prediction. In Proceedings of the 27th annual international symposium on Microarchitecture, pages 196-206. ACM, 1994.

[19] Xilinx. Spartan-6 Family Overview, 2011.

[20] Xilinx. Virtex-6 Family Overview, 2015. 